\title{
Effect of pH on Optical Properties of Graphene Oxide Quantum Dots
}

Ahmad Zia Sherzad $^{\mathrm{a}}$, Hakimeh Zare ${ }^{\mathrm{a} * *}$, Zahra Shahedi $^{\mathrm{a}}$, Fatemeh Ostovari ${ }^{\mathrm{a}}$, Yousef Fazaeli ${ }^{\mathrm{b}}$, and Zeinab Pourghobadi ${ }^{\mathrm{c}}$

\author{
${ }^{\text {a}}$ Physics Department, Faculty of Science, Yazd University, Yazd, Iran \\ ${ }^{b}$ Radiation Application Research School, Nuclear Science and Technology Research Institute \\ (NSTRI), Karaj, Iran \\ ${ }^{c}$ Department of Chemistry, Khorramabad Branch, Islamic Azad University, Khorramabad, \\ Iran \\ *Corresponding author Email: hzare@yazd.ac.ir
}

\begin{abstract}
Regular paper: Received: May. 27, 2020, Revised: Jul. 23, 2020, Accepted: Aug. 24, 2020, Available Online: Aug. 26, 2020, DOI: 10.29252/ijop.14.2.135
\end{abstract}

\begin{abstract}
Luminescent graphene oxide quantum dots (GOQDs) have attracted tremendous attention from scientists in chemistry, materials, biology, and physics science. They have specific properties such as low cytotoxicity, excellent electrochemical and optical properties, resistance to photobleaching, and good stability. In this study, GOQDs were synthesized using a simple and straightforward methodology. The synthesized GOQDs were characterized by Fourier Transform Infrared (FTIR) analyzer, ultraviolet-visible spectrophotometry (UV-VIS) absorption, Photoluminescence (PL) spectroscopy, and transmission electron microscopy (TEM) analyses. Then, optical properties of GOQDs such as absorption and luminescence with various $\mathrm{pH}$ values were investigated. GOQDs show absorption in the ultraviolet (UV) region and their position of photoluminescence peak is independent of $\mathrm{pH}$ value. The average size of QDs is less than $5 \mathrm{~nm}$, as revealed by TEM. The GOQDs show green luminescence under $U V$ irradiation $(360 \mathrm{~nm})$.
\end{abstract}

KEYWORDS: Graphene Oxide, Quantum Dots, $\mathrm{pH}$, Photoluminescence, UV-Vis.

\section{I.INTRODUCTION}

Quantum dots are called semiconductor nanocrystals with size of about 1 to $10 \mathrm{~nm}$ [13], which are widely used in light-emitting diodes (LEDs), lasers, environmental displays, electronic displays, and solar cells due to their unique electro-photo properties depending on their size [4-7]. However, due to the high cost and toxicity of quantum dots based on, their use in industry has been limited [6]. In recent decades, as a cost-effective and promising alternative, graphene quantum dots (GQDs), graphene oxide quantum dots (GOQDs), and carbon quantum dots (CQDs) have emerged as a new class of nanomaterials [8, 9]. Graphene oxide (GO) is a modern, single-layer structure of the well-known material of graphite oxide. Graphite oxide is not a substance found in nature and is artificially produced as a single layer of carbon atoms with a sp2 bond in a honeycomb network [10-12]. By reducing the particle size of graphene oxides to a multinanometer scale, the optical properties and hydrophobicity of graphene oxide can be adjusted. These particles are called "graphene oxide quantum dots." Due to their very low toxicity, hydrophobicity, and high optical efficiency, GOQDs promise extensive medicine applications [13-15].

So far, many methods have been reported to synthesis of GO and GOQDs, that are usually requiring a long reaction time. Graphene derivatives are generally produced via two methods: "bottom-up" and "top-down". The first method uses small molecular precursors such as citrate, carbohydrates, glucosamine, ascorbic acid, saccharides, via combustion or, solvothermal process to make graphene derivatives [16-21]. The second method (top- 
down) usually breaks down more abundant carbon sources by means like electrochemical oxidation, laser ablation, and arc discharge [22-28]. Moreover, this should be mentioned that the above methods have risk of direct exposure to flammable carbon sources, strong oxidants and, strong acids, which may explode or burn, the synthesis process is quite tedious and time-consuming. In this research, we will report a secure and easy one-pot synthesis method using citric acid (CA) as the starting material for synthesis GOQDs. This method is quite simple and does not require tedious and Time processes.

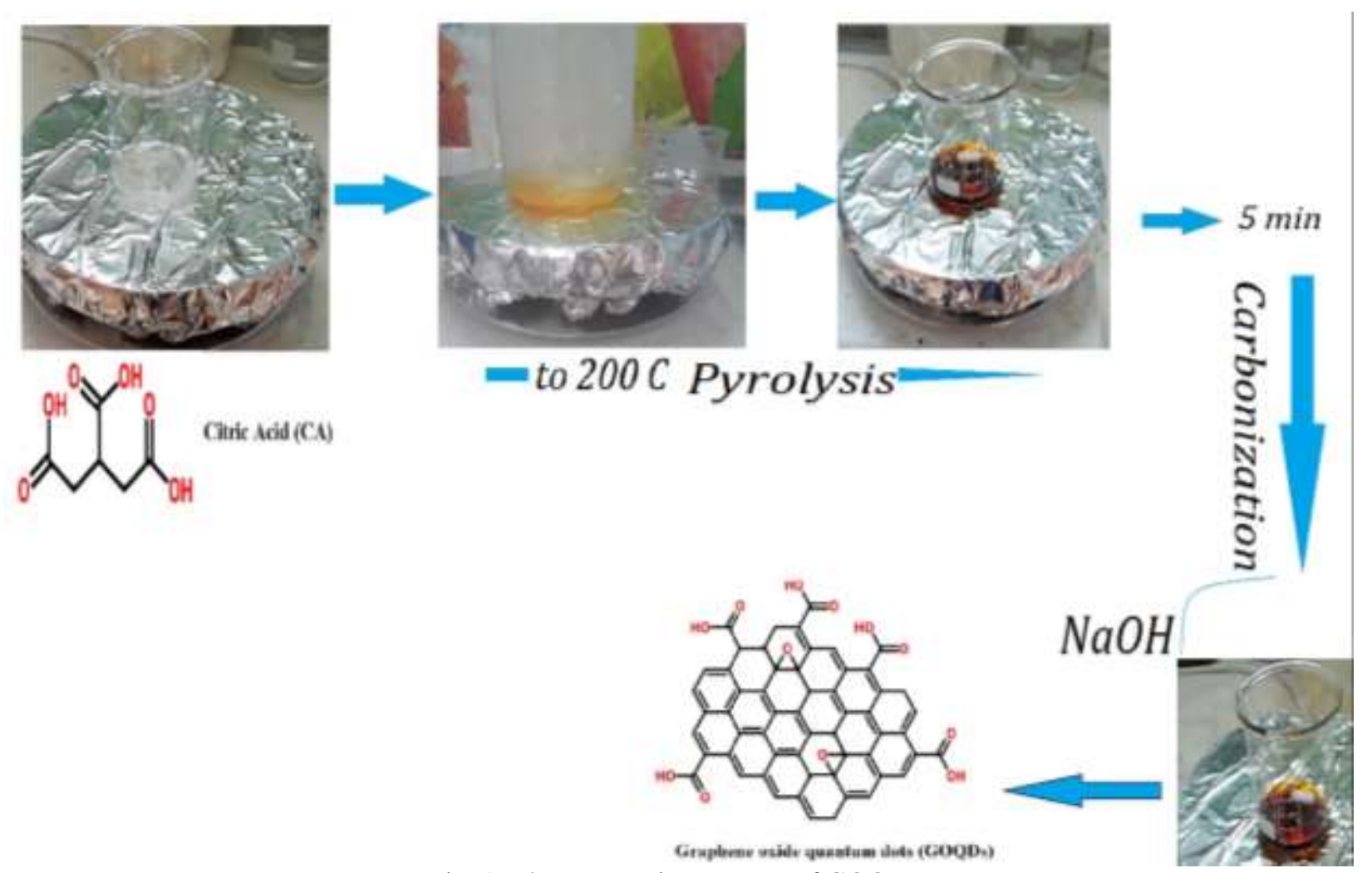

Fig. 1. The preparation process of GOQDs.

Also, it has a much higher level of safety than other synthesis methods. In continuation, we study and analyze GOQDs at different $\mathrm{pHs}$ using transmission electron microscopy (TEM), infrared spectroscopy (FT-IR), ultra violet-visible (UV-Vis) absorption, and photoluminescence (PL) spectroscopy.

\section{EXPERIMENTAL SECTION}

\section{A. Materials}

For synthesis of GOQDs, CA powder $\left(\mathrm{C}_{6} \mathrm{H}_{8} \mathrm{O}_{7}, 99 \%\right)$, sodium hydroxide $(\mathrm{NaOH}$, $97 \%)$ and ethanol $\left(\mathrm{C}_{2} \mathrm{H}_{5} \mathrm{OH}, 99 \%\right)$ were bought from Merck. The chemicals were all analytically pure and used without purification. Deionized (DI) water at room temperature was used for diluting samples to the ideal concentration throughout the experiment and washing.

\section{B. Synthesis of GOQDs}

GOQDs were synthesized using pyrolyzing citric acid (CA). Briefly, 4 gr of CA powder heated to $200{ }^{\circ} \mathrm{C}$ using a heater stirrer for 5 min. During the heating process, the CA powder was first melted, and in the meantime, the color of the solution changed from colorless to yellow and eventually to dark brown within 1-10 min. Then, 2 gr of $\mathrm{NaOH}$ powder was dissolved in $50 \mathrm{ml}$ of DI water. The solution of $\mathrm{NaOH}$ was added dropwise in the melted solution of $\mathrm{CA}$ at $35^{\circ} \mathrm{C}$, to prepare the solutions of different $\mathrm{pH}$ ranging from 7 to 10. The effect of different $\mathrm{pHs}$ on the yield optical properties of the GQDs was studied in detail. Finally, the synthesized GOQDs solution was precipitated with ethanol, separated by centrifugation, and at last 
dispersed in ultrapure water. The synthesis process is shown in Fig. 1.

\section{Characterization}

Characterizations of the synthesized GOQDs were conducted using TEM, FT-IR, UV-Vis, and, PL analyses. The TEM image of the GOQDs was acquired on a JEOL, JEM-2100F, $200 \mathrm{KV}$ electron source. Fourier Transform Infrared (FTIR) analyzer spectrum in region $200-4000 \mathrm{~cm}^{-1}$ were restored on an Equinox 55 FTIR spectrometer using Potassium bromide $(\mathrm{KBr})$ pellets. Absorption spectra were recorded in area $200-900 \mathrm{~nm}$ by using Perkin Elmer $2500 \quad$ UV-Vis spectrophotometer. The PL spectra were measured with G9800A Agilent, under ambient conditions.

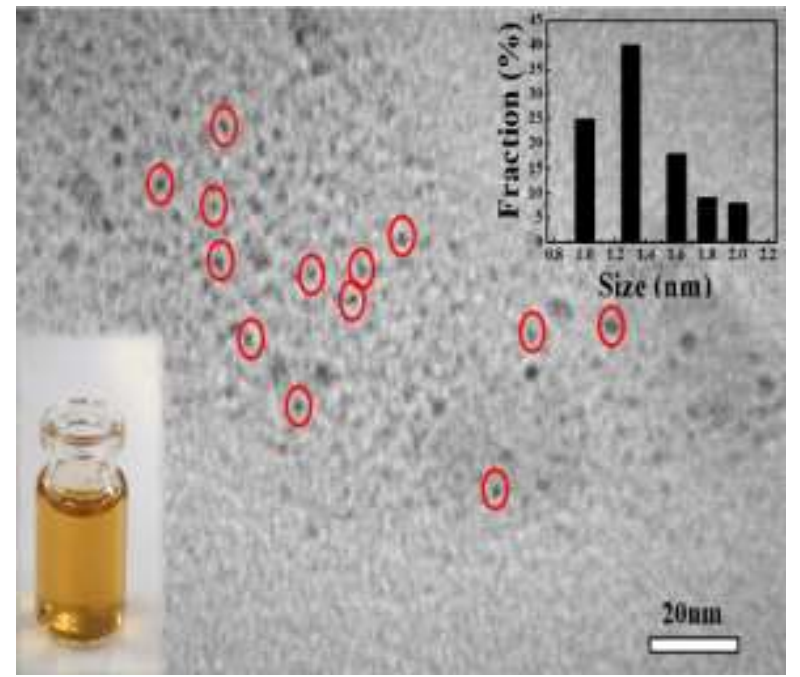

Fig. 2. TEM image and the corresponding size distribution curves of GOQDs.

\section{III.RESULTS AND DISCUSSION}

In our paper, the GOQDs are prepared using CA powder by $5 \mathrm{~min}$ of heating at $200^{\circ} \mathrm{C}$. The TEM image and the corresponding size distribution plot of the GOQDs for sample with $\mathrm{pH}=10$ are shown in Fig. 2. Results reveal that QDs have quasi-spherical shapes with a nearly monodisperse size distribution. The mean diameters of the GOQDs is about $2 \mathrm{~nm}$. Due to the presence of $-\mathrm{COOH}$ groups at the edges thereof, the particles are well dispersed, which can be seen in the Fig. 2.
The synthesized GOQDs were characterized by FTIR analysis. Fig. 3 shows the FTIR spectrum of QDs in the range of 400-4000 $\mathrm{cm}^{-1}$ for $\mathrm{pH}=10$. The FTIR spectrum of GOQDs shows the presence of $\mathrm{C}-\mathrm{O}(\mathrm{vC}-\mathrm{O}$ at 1048 , and $\left.1096 \mathrm{~cm}^{-1}\right), \mathrm{C}=\mathrm{O}$ in carboxylic acid, $\mathrm{C}-\mathrm{OH}\left(\mathrm{vC}-\mathrm{OH}\right.$ at $\left.1399 \mathrm{~cm}^{-1}\right)$, and carbonyl moieties (vC $=\mathrm{O}$ at 1565 and $1737 \mathrm{~cm}-1)$, band around $2972 \mathrm{~cm}^{-1}$ due to both sp2 and sp3 C$\mathrm{H}$ modes, and the $\mathrm{O}-\mathrm{H}$ band (broadband around $3420 \mathrm{~cm}^{-1}$ ) [29]. According to the articles, $\mathrm{pH}$ does not have an effect on reducing and increasing the grafts and functional groups of GOQDs and may only cause shifts [30].

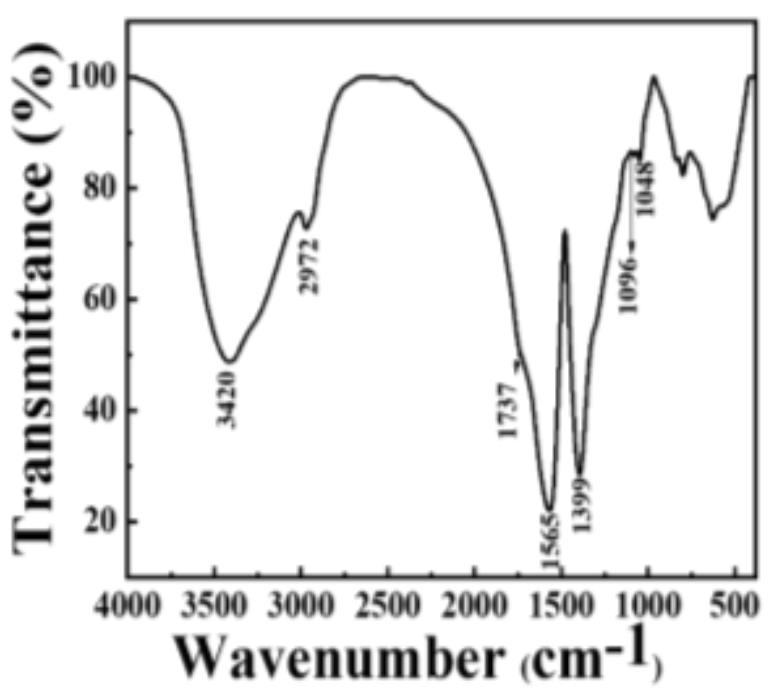

Fig. 3. FTIR spectrum of GOQDs.

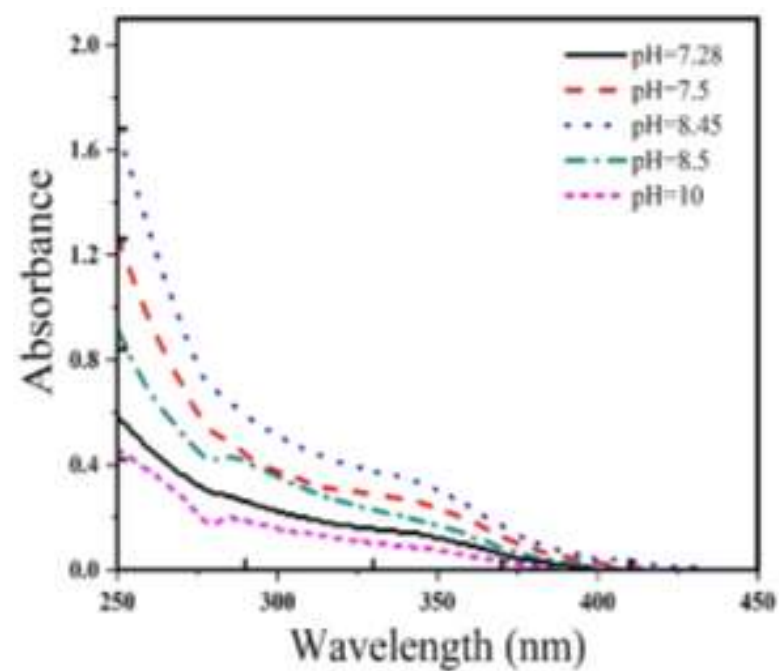

Fig. 4. Absorption spectra of GOQDs in the various $\mathrm{pH}$ values. 
Figure 4 shows the absorption spectra of GOQDs with various $\mathrm{pH}$ values in the aqueous solution. For GOQDs in the Fig. 4, as can be seen, GOQDs show the broad absorption in the UV region with a tail that extends to the visible range. The GOQDs show two shoulder peaks at 210-230 and $340 \mathrm{~nm}$. The absorption peaks at $210-230 \mathrm{~nm}$ can be ascribed to $\pi-\pi^{*}$

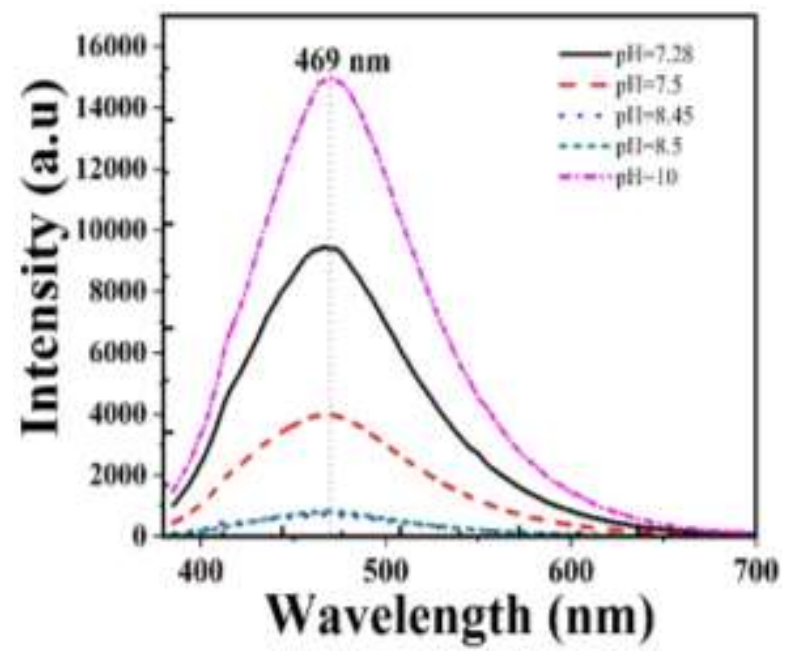

Fig. 5. Fluorescence spectra of GOQDs in the various $\mathrm{pH}$ values.

Electronic transitions of the aromatic $\mathrm{C}-\mathrm{C}$ bonds in their structure. The absorption shoulder peaks at $340 \mathrm{~nm}$ can be related to the functional groups on their edges, correctly, $\pi^{*}-\mathrm{n}$ transitions of the $\mathrm{C}=\mathrm{O}$ bonds [29]. The GOQDs show a peak in the range of 286 to $300 \mathrm{~nm}$, due to the absorption of graphite structure in water, similar to GO. The absorption intensity in this range for different $\mathrm{pHs}$ is increasing and decreasing. Increased adsorption indicates that the electronic connections will be restored after rupture [31]. The UV-Vis spectra proved that on increasing the $\mathrm{pH}$ to 8.5 , the absorption peaks become broader which caused the increase of distribution of particle size along with the $\mathrm{pH}$. After this $\mathrm{pH}$, the intensity of the peaks decreases up to 10 .

Figure 5 presents the room temperature PL spectra of as-prepared GOQDs with various $\mathrm{pH}$ values. PL spectra are recorded with an excitation wavelength of $360 \mathrm{~nm}$. The PL spectra of the most carbon materials depends on the wavelength of their excitation. More and more cases have emerged with excited wavelength that are independent of emission position and can be attributed to their surface chemistry and uniform size. For the all five samples, the emission peaks do not shift in the various $\mathrm{pH}$ values, but its intensity decreases by increasing $\mathrm{pH}$ from 7.3 to 8.5 and increases with a $\mathrm{pH}$ of 10 . The emission peak position of all samples was located at $469 \mathrm{~nm}$ and the highest photoluminescence intensity related to GOQDs with $\mathrm{pH}=10$. This immutability in the peak position of the PL at different $\mathrm{pHs}$ indicates the independence of GOQDs relative to the $\mathrm{pH}$. The primary source of the emission peak position of these materials may be free zigzag modes with a triple carbon state [32]. By increasing the $\mathrm{pH}$, the increasing number of hydroxyl groups gets adhered to the QDs and resulting in an increase in the size of the QDs and lower PL. But, it may be attributed to the fact that in the case of $\mathrm{pH}=10$, the presence of hydroxyl groups impeded the aggregation of the carbon nanoparticles and caused an increase in the size of the nanoparticles and PL. Thus, the $\mathrm{pH}$ of the CA solution played a vital role in the synthesis of GOQDs and their optical properties.

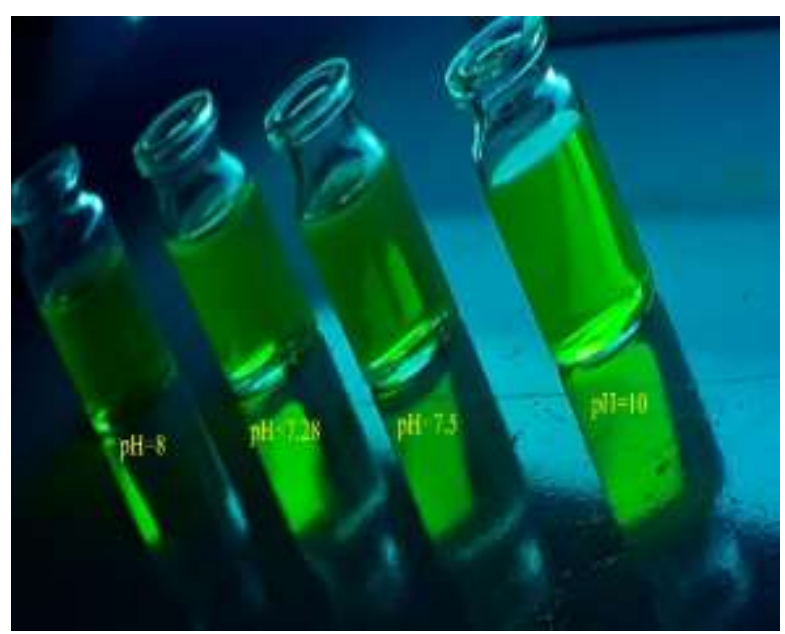

Fig. 6. Photographs of GOQDs under UV light $(\lambda=360 \mathrm{~nm})$ in various $\mathrm{pH}$ values.

Figure 6 indicates that the solutions of GOQDs are transparent under UV irradiation, and large particles are not observed in them. Therefore, these QDs are well-dispersed in the aqueous phase. The as-prepared GOQDs solutions emit bright green color under UV illumination. The 
emission colors of these GOQDs are consistent with the corresponding PL spectra in the Fig. 5. Also, the emission color of all samples is unchanged in various $\mathrm{pH}$ values that compatible with the results of PL spectra in Fig. 5. The high transparency of the solutions indicates that the size of the QDs is less than $10 \mathrm{~nm}$.

\section{IV.CONCLUSION}

GOQDs were synthesized in the various $\mathrm{pH}$ values by pyrolyzing $\mathrm{CA}$. Then, the effect of $\mathrm{pH}$ on physical and optical properties of synthesized QDs was investigated by FTIR, TEM, and optical spectroscopy. The FTIR analysis of the samples did not change with $\mathrm{pH}$. TEM analysis showed that the particles size diameter was almost the same and less than $5 \mathrm{~nm}$. The UV-Vis spectra of all samples showed two absorption peaks, $n-\pi *$ and $\pi-\pi *$ transitions, related to $\mathrm{C}=\mathrm{O}$ and $\mathrm{C}=\mathrm{C}$ bonds, which are observed in different pHs. The PL spectra of samples showed that the PL peak position did not change in the various $\mathrm{pH}$ values, indicating the independence of the PL of the GOQDs from the $\mathrm{pH}$.

\section{REFERENCES}

[1] D. Pan, J. Zhang, Z. Li, and M. Wu, "Hydrothermal route for cutting graphene sheets into blue-luminescent graphene quantum dots," Adv. Mater. Vol. 22, pp. 734$738,2010$.

[2] H. Cheng, Y. Zhao, Y. Fan, X. Xie, L. Qu, and G. Shi, "Graphene-quantum-dot assembled nanotubes: a new platform for efficient Raman enhancement," Acs Nano, Vol. 6, pp. 22372244, 2012.

[3] J. Shen, Y. Zhu, X. Yang, and C. Li, "Graphene quantum dots: emergent nanolights for bioimaging, sensors, catalysis and photovoltaic devices," Chem Comm. Vol. 48, pp. 3686-3699, 2012.

[4] Ch. X. Guo, Y. Dong, H.B. Yang, and Ch. M. $\mathrm{Li}$ "Graphene quantum dots as a green sensitizer to functionalize $\mathrm{ZnO}$ nanowire arrays on F-doped $\mathrm{SnO}_{2}$ glass for enhanced photoelectrochemical water splitting," Adv. Energy Mater. Vol. 3, pp. 997-1003, 2013.
[5] L. Chen, Ch.X. Guo, Q. Zhang, Y. Lei, J. Xie, Sh. Ee, G. Guai, Q. Song, and Ch.M. Li, "Graphene quantum-dot-doped polypyrrole counter electrode for high-performance dyesensitized solar cells," ACS Appl. Mater. Interfaces, Vol. 5, pp. 2047-2052, (2013.

[6] Y. Xu, X. Li, G. Hu, T. Wu, Y. Luo, L. Sun, T. Tang, J. Wen, H. Wang, and M. Li, "Graphene oxide quantum dot-derived nitrogen-enriched hybrid graphene nanosheets by simple photochemical doping for highperformance supercapacitors," Appl. Surf. Sci. Vol. 422, pp. 847-855, 2017.

[7] J. Sun, S. Yang, Zh. Wang, H. Shen, T. Xu, L. Sun, H. Li, W. Chen, X. Jiang, G. Ding, Zh. Kang, X. Xie, and M. Jiang, "Ultra-High Quantum Yield of Graphene Quantum Dots: Aromatic-Nitrogen Doping and Photoluminescence Mechanism," Particle \& Particle Systems Characterization, Vol. 32, pp. 434-440, 2015.

[8] Y. Xu, H. Bai, G. Lu, Ch. Li, and G. Shi, "Flexible graphene films via the filtration of water-soluble noncovalent functionalized graphene sheets," J. Am. Chem. Soc. Vol. 130, pp. 5856-5857, 2008.

[9] F. Bonaccorso, Z. Sun, T.A. Hasan, and A.C. Ferrari, "Graphene photonics and optoelectronics," Nat. Photonics, Vol. 4, pp. 611-622, 2010.

[10]D.V. Kosynkin, A.L. Higginbotham, A. Sinitskii, J.R. Lomeda, A. Dimiev, B.K. Price, and J.M. Tour, "Longitudinal unzipping of carbon nanotubes to form graphene nanoribbons," Nature, Vol. 458, pp. 872-876, 2009.

[11] A.K. Geim, "Graphene: status and prospects." Science, Vol. 324, pp. 1530-1534, 2009.

[12] M.I. Katsnelson, "Graphene: carbon in two dimensions," Mater. Today, Vol. 10, pp. 2027, 2007.

[13] Y. Shao, J. Wang, H. Wu, J. Liu, I.A. Aksay, and Y. Lina, "Graphene based electrochemical sensors and biosensors: a review," Electroanalysis: An International J. Devoted to Fundamental and Practical Aspects of Electroanalysis, Vol. 22, pp. 1027-1036, 2010.

[14] X. Huang, X. Qi, F. Boey, and H. Zhang, "Graphene-based composites," Chem. Soc. Rev. Vol. 41, pp. 666-686, 2012. 
[15]Z. Ma, S. Dou, A. Shen, L. Tao, L. Dai, and S. Wang, "Sulfur-Doped Graphene Derived from Cycled Lithium-Sulfur Batteries as a Metal-Free Electrocatalyst for the Oxygen Reduction Reaction," Angew. Chem. Vol. 54, pp. 1888-1892, 2015.

[16] Sh. Zhu, Q. Meng, L. Wang, J. Zhang, Y. Song, H. Jin, K. Zhang, H. Sun, H. Wang, and B. Yang, "Highly photoluminescent carbon dots for multicolor patterning, sensors, and bioimaging," Angew. Chem. Vol. 52, pp. 3953-3957, 2013.

[17]H. Peng and J. Travas-Sejdic, "Simple aqueous solution route to luminescent carbogenic dots from carbohydrates," Chem. Mater. Vol. 21, pp. 5563-5565, 2009.

[18] S. Liu, N. Zhao, Z. Cheng, and H. Liu, "Amino-functionalized green fluorescent carbon dots as surface energy transfer biosensors for hyaluronidase," Nanoscale, Vol. 7, pp. 6836-6842, 2015.

[19] S. Gómez-de Pedro, A. Salinas-Castillo, M. Ariza-Avidad, A. Lapresta-Fernández, C. Sánchez-González, C.S. Martínez-Cisneros, M. Puyol, L.F. Capitan-Vallvey, J. AlonsoChamarro, "Microsystem-assisted synthesis of carbon dots with fluorescent and colorimetric properties for $\mathrm{pH}$ detection," Nanoscale, Vol. 6, pp. 6018-6024, 2014.

[20]L. Tang, R. Ji, X. Cao, J. Lin, H. Jiang, X. Li, K.S. Teng, Ch. Man Luk, S. Zeng, J. Hao, and Sh. Ping Lau, "Deep ultraviolet photoluminescence of water-soluble selfpassivated graphene quantum dots," ACS Nano, Vol. 6, pp. 5102-5110, 2012.

[21]S.Y. Lim, W. Shen, and Z. Gao, "Carbon quantum dots and their applications," Chem. Soc. Rev. Vol. 44, pp. 362-381, 2015.

[22] H. Li, Z. Kang, Y. Liu, and S.T. Lee, "Carbon nanodots: synthesis, properties and applications," J. Mater. Chem. Vol. 22, pp. 24230-24253, 2012.

[23]H. Liu, T. Ye, and C. Mao, "Fluorescent carbon nanoparticles derived from candle soot," Angew. Chem. Vol. 46, pp. 6473-6475, 2007.

[24] Y. Dong, C. Chen, X. Zheng, L. Gao, Zh. Cui, H. Yang, Ch. Guo, Y. Chi, and Ch. Ming Li, "One-step and high yield simultaneous preparation of single-and multi-layer graphene quantum dots from CX-72 carbon black," J. Mater. Chem. Vol. 22, pp. 8764-8766, 2012.

[25]H. Yuan, D. Li, Y. Liu, X. Xu, and C. Xiong, "Nitrogen-doped carbon dots from plant cytoplasm as selective and sensitive fluorescent probes for detecting p-nitroaniline in both aqueous and soil systems," Analyst. Vol. 140, pp. 1428-1431, 2015.

[26] L. Zheng, Y. Chi, Y. Dong, J. Lin, and B. Wang, "Electrochemiluminescence of watersoluble carbon nanocrystals released electrochemically from graphite," J. Am. Chem. Soc. Vol. 131, pp. 4564-4565, 2009.

[27] Y.-P. Sun, B. Zhou, Y. Lin, W. Wang, K. A. Shiral Fernando, P. Pathak, M. Jaouad Meziani, B. A. Harruff, X. Wang, H. Wang, P. G. Luo, H. Yang, M. Erkan Kose, B. Chen, L. Monica Veca, and S.-Y. Xie, "Quantum-sized carbon dots for bright and colorful photoluminescence," J. Am. Chem. Soc. Vol. 128, pp. 7756-7757, 2006.

[28]M. Bacon, S.J. Bradley, and T. Nann, "Graphene quantum dots," Particle \& Particle Systems Characterization, Vol. 31, pp. 415428, 2014.

[29] J.M. Yuan, R. Zhao, Z.J. Wu, W. Li, and X.G. Yang "graphene oxide quantum dots exfoliated from carbon fibers by microwave irradiation: two photoluminescence centers and self-assembly behavior," Small, Vol. 14, pp. 1703714, 2018.

[30]Ch. Ran, M. Wang, W. Gao, Zh. Yang, J. Shao, J. Deng, and X. Song, "A general route to enhance the fluorescence of graphene quantum dots by Ag nanoparticles," RSC advances, Vol. 4, pp. 21772-21776, 2014.

[31] Y. Feng, J. Zhao, X. Yan, F. Tang, and Q. Xue, "Enhancement in the fluorescence of graphene quantum dots by hydrazine hydrate reduction," Carbon, Vol. 66, pp. 334-339, 2014.

[32]A. Mehta, E.J. Nelson, S.M. Webb, and J.K. Holt, "The interaction of bromide ions with graphitic materials," Adv. Mater. Vol. 21, pp. 102-106, 2009. 


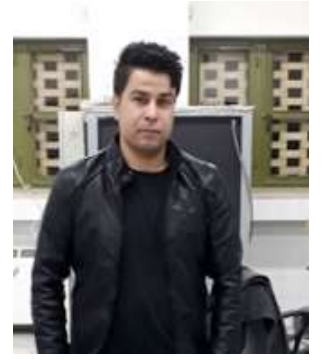

Ahmad Zia Sherzad received his M.Sc. in solid State Physics, from Yazd University, Yazd, Iran, in 2020. His main interests include nano-physics, and naobiophysics.

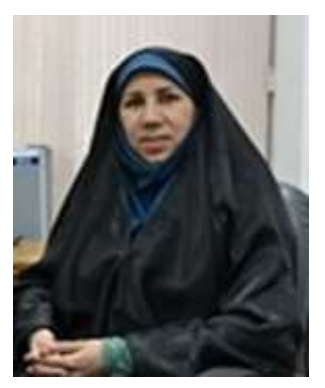

Hakimeh Zare received her Ph.D. in Nanotechnology from the Sharif University of Technology, Tehran, Iran., in 2015. Dr. Zare has been a tenured member of Physics Department, Yazd University, Yazd, Iran, ever since. Her main interests include nanooptoelectronic, nano-physics, and naobiophysics.

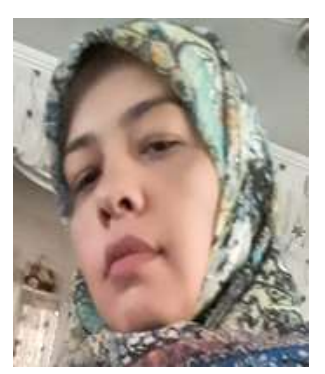

Zahra Shahedi received her Ph.D. in Physics from the Arak University, Arak, Iran. in 2017. Dr. Zare Her main interests include nanooptoelectronic, nano-physics, and naobiophysics.

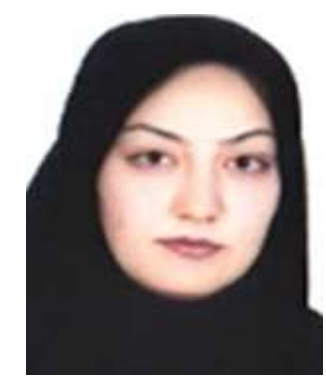

Fatemeh Ostovari received her Ph.D. in Physics from the Tarbiat Modares University, Tehran, Iran., in 2014. Dr. Ostovari has been a tenured member of Physics Department, Yazd University, Yazd, Iran, ever since. Her main interests include nanooptoelectronic, nanophysics, and naobiophysics.

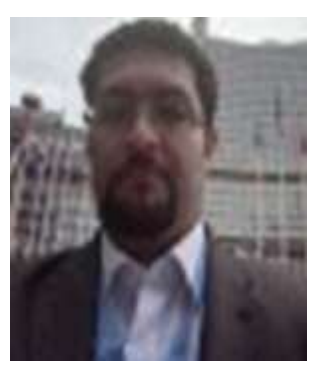

Yousef Fazaeli is associate professor of chemistry at the Nuclear Science and Technology Research Institute (NSTRI). Dr. Fazaeli received his Ph.D. in Inorganic Chemistry from the SBU (Tehran, Iran) in 2012. He joined the NSTRI in 2008, where he served as head of the Radiolabeling laboratories. His research interests are in the field of radiochemistry, with a special focus on radioisotope and radiopharmaceutical production with cyclotron and research reactors. He has been involved in many collaborative research projects financed by various institutions including the International Atomic Energy Agency (IAEA). $\mathrm{He}$ is the author /(co-) author of 62 journal papers and 90 publications in national and international conference proceedings. 


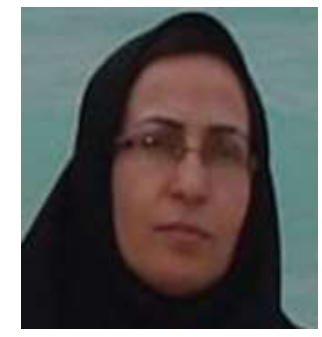

Zeinab Pourghobadi received her PhD degree in analytical chemistry in 2014 from Department of Chemistry, Arak Branch,
Islamic Azad University, Arak, Iran. At the present Dr. Pourghobadi is member of Department of Chemistry, Khorramabad Branch, Islamic Azad University, Khorramabad, Iran. Her main interests cover electrochemical, electrochemical sensors, optical and biosensors. 